\title{
Rethinking social marketing: Towards a sociality of consumption
}

Purpose: The purpose of this paper is to explore how members of an online alcohol reduction community learn, construct and engage in alcohol reduction consumption consistencies.

Methodology: Textual and visual material, primarily blogs, from 15 individuals participating in the online community of Hello Sunday Morning were collected and analysed. Participants engaged in a series of in-depth interviews to gain a perspective into alcohol reduction action, activities and interactions.

Findings: The findings indicate learning of new alcohol reduction consumption consistencies occurs through three modes or learning infrastructures: engagement, imagination and alignment enabling a collective sense of connection in the creation of new alcohol-related rituals and traditions, competency of practices and transmission of values and norms beyond the community.

Research implications: The results underscore the need for social marketers to recognise learning of alcohol reduction behaviour is continually negotiated and dynamically engendered through socially reproduced conditions, responses and relationships.

Originality: This study contributes to the transformational potential of social marketing situating behaviour change as a social interaction between actors within a dynamic market system.

Key words: responsible drinking, community of practice, habits, behaviour change, social marketing

Article classification: research paper

Citation for this paper is:

Fry, M.L. (2014), 'Rethinking social marketing: Towards a sociality of consumption', Journal of Social Marketing, 4(3): 210-222. 


\section{Rethinking social marketing: Towards a sociality of consumption}

\section{Introduction}

Behaviour change is accepted as at the core, and the bottom line, of social marketing (Andreasen, 1995). Nonetheless, current scholarship contends social marketing is narrowly fixated on behavioural outputs as the key measure of program success (French et al., 2012; Donovan, 2011). Consequently attention is directed to viewing the social phenomena under investigation at an individual level premised on the assumption individuals are responsible for their own health quality (Szmigin et al., 2011; Wymer, 2011). This purview of social marketing neglects rather than considers the wider social forces impacting behaviour, and that behaviour change is a relational interaction between individuals within social contexts (Wymer, 2011; Szmigin et al., 2011). Lefebvre (2012) challenges the state of play of social marketing suggesting the discipline has become myopic in its continued application of marketing principles to influence individual behaviour rather than attempting to shape markets.

Calling for a broadening of the scope of social marketing, critics advocate for greater inclusion of contemporary marketing insights into social marketing planning and development (Lefebvre, 2012, 2011; French, et al., 2012; Donovan, 2011). A market systems approach urges social marketers to think beyond individual behaviour towards a conceptualisation of behaviours and behaviour change as operating within a dynamic, evolving market. As Lefebvre (2012) notes, viewing behaviour change as 'markets of behaviours' represents 'out of the box' thinking. Critically, this viewpoint brings to the forefront the 'social' in social marketing. 
This paper contributes to the transformational potential of social marketing situating alcohol behaviour change as operating as a social interaction between actors within a dynamic market system. Viewing behaviour change as a socially entwined practice between individuals and individuals as active participants in the behaviour change process, the purpose of this paper is to investigate and explain how members of an online alcohol reduction community - Hello Sunday Morning - learn, construct and engage in new alcohol reduction consumption consistencies. To date social marketing for alcohol has largely focussed on intervention effectiveness with little attention directed towards understanding individual's transitioning from risky to less risky behaviour.

To achieve this, Wenger’s (1998, 2000) Communities of Practice (CoP) Theory is applied to investigate and explain individual's learning of intentional-alternate alcohol reduction consumption consistencies. Prior to discussing the CoP approach as related to social marketing the following section elaborates on the potential role of habit formation, or more importantly disruption of habits and creation of new habits, as central in the learning of new consumption consistencies. Study findings explicating HSMers learning of new alcohol consumption consistencies and implications for social marketing are then discussed.

\section{New consumption consistencies \& the sociality of habits}

Consumer culture theory suggests individuals, through experiences, actively transform symbolic meanings from consumer culture to further their identity position and lifestyle goals, which are continually fashioned and co-produced with other partners within the social space (Shanker et al., 2009). Extending this viewpoint to social marketing, Peattie and Peattie (2009) suggest the effectiveness of consumption reduction programs in overcoming deeply engrained behaviours rests on the extent to which new consumption reduction behaviours become more widely appealing in society, and viewed as normal. 
Kruglanski et al’s (2002) theory of goal systems proposes goal directed behaviours for performing and not performing certain actions belong to two separate decision-making systems with distinct sets of cognitive reasoning for each alternative, suggesting that decision making processes and the pathways to action and inaction are significantly different and psychologically distinct (Richetin et al., 2011). As such, intentions for drinking to get drunk (e.g. to have fun, socialise with friends, get drunk) provide quite separate reasons than those for drinking sensibly (e.g. improve health, look good, not rely on alcohol for a good time). Goal systems theory opens a space for considering responsible drinking as a consumption entity that is more than simply diametrically oppositional to that of intoxication (Fry, 2011). Considering responsible drinking as a viable consumption entity, Wood and Neal (2007) assert creation of new consumption consistencies is dependent on disrupting environmental factors that automatically cue habit performance. Overtime, habit performances, as recurring performance acts, become increasingly triggered by environmental cues, internal states (e.g. moods) and through interactions with others (Verplanken and Wood, 2006). Habits by nature require little, if any, cognitive input from consumer's intentions or decisions to act. It is the dependent nature of habit formation involving the creation of new associations in memory between actions and the automatic cueing of behaviour by stable performance circumstances that Verplanken and Wood (2006:91) argue represents an ‘important point of vulnerability’. Habits represent a barrier to information use as they are not easily changed through persuasive appeals, yet they can be influenced through changes in environmental circumstances or modifying the context in which behaviours are performed (Wood and Neal, 2007).

As such, disrupting environmental cues that trigger and maintain specific performances provides social marketers with the opportunity to not only challenge habitual mind-sets, but also put in place strategies to impair the automatic cueing of well-practiced responses 
(Verplanken and Wood, 2006). Unfreezing existing behavioural patterns, thus opening habits to change, allows for the creation of new information, actions, experiences, and behaviours through the formation of new consumption consistencies which involve new patterns and habit performances. Jackson (2005) maintains unfreezing existing behavioural patterns needs to occur at the community level where group members actively support those involved in negotiating change. Harnessing this perspective, any success in positioning responsible drinking as a valid consumption entity is underpinned by recognising behaviour change as a social proposition.

\section{Consumption communities as spaces enabling intentional alternate behaviour}

In the marketing literature the concept of consumer brand communities is well established (Schau et al., 2009; Muniz and O’Guinn, 2001). Yet, leveraging community as an agent of social change in alcohol-related social marketing remains at the margin (Szmigin et al., 2011; Fry 2010). Virtual communities of consumption represent collectives where people gather together beyond traditional social or geographic boundaries, to learn, form and reinforce relationships on a casual basis on any given field of interest (Kozinets, 1999). Hagel and Armstrong (1997) emphasize the relationship building potential of virtual communities as they provide, for people with similar interests, an opportunity to come together, unrestrained by time and space, to meet some of their social and commercial needs.

For Wenger (1998, 2000) learning is a social phenomenon and is defined as the 'interplay between social competence and personal experience’ (Wenger, 2000, p. 227). Viewing learning as an active entity, Wenger (2000) emphasises learning as a relational, dynamic, two-way interaction between people and the social learning systems in which they participate. Communities of practice (CoP) facilitate knowledge sharing as it is within a community that competence and experience converge enabling learning to take place. 
Members need to contribute knowledge, but must also have knowledge gaps to be filled by the community, yet knowledge can only be harnessed through practice (Brown and Duguid, 1991). It is the sharing of practice experiences within the CoP that enables participants to internalize tacit knowledge with sharing activities simultaneously encouraging member participation, knowledge creation, accumulation and diffusion (Ardichvilli et al., 2003). CoPs evolve from things individual's value or rate as important and, as such, represent situated learning where individuals develop practices, norms, values and relationships appropriate to the community (Handley et al., 2006).

Wenger (2000) identifies three infrastructures or modes for learning essential for productive CoPs: engagement, imagination and alignment. Without engagement communities of practice would not exist. Engagement represents the connectivity between members; the mutual agreement in taking responsibility for learning how to enact responsible drinking and is the active involvement in the mutual processes of negotiating meaning. Participation is crucial to engagement as without participation knowledge is not created, therefore does not evolve.

Imagination refers to the construction and sense making in developing new competencies within the community alongside others. Imagination enables community members to share a collective consciousness in the doing of alcohol reduction, and locating their engagement in a broader system other than the self. Through engagement in the collective imagination is a process of expanding the self through learning to engage, create and improvise alcohol reduction practices. A community of practice, according to Wenger (1998) needs practices which solidify community purpose and membership through processes of reification, with reification referring to a wide range of process contributing to the sense-making and creating of responsible drinking practices. In practical terms imagination is referring to opportunities 
for learners to think out of the box, to break from the norm, to journey outside the dominant norm.

Alignment represents common patterns of action and diffusion of ideals, practices and identity within, and more importantly beyond the boundaries of the group. As Wenger (2000) notes alignment requires sharable artefacts, creation and adoption of broader discourses surrounding responsible drinking within society, and moving towards a discursive consciousness of what is responsible drinking, how to perform responsible drinking and acceptance of responsible drinking as a consumption entity.

While these modes of learning are distinct, they operate in concert. Neither alignment nor engagement provides capability for grounded sense-making. Imagination is required for reflexivity of responsible drinking sense making, yet needs to be grounded by alignment and engagement. Thus, $\mathrm{CoP}$ as social containers that bring people together leverage the complementarity of the three learning modes to facilitate a sense of joint enterprise, mutual engagement and a shared repertoire of resources (Wenger, 2000).

\section{Method}

Hello Sunday Morning (HSM) (www.hellosundaymorning.org) an online alcohol reduction consumption community is the context of investigation. Operating independently of Australia’s public health alcohol prevention initiatives HSM morphed from a personal journey of one person - Chris Raine - who decided to give up drinking in 2009 and blog about his experiences. Named in reference to not waking up to a Sunday Morning with a hangover Chris's first person introspective contributed valuable insight into the process, emotions, sociality and psychological responses of living and socialising without alcohol. During the year of 'giving up' the blog attracted over 1,000 followers (Hamley and Carah, 
2012). Recognising the limits of government-based alcohol strategies Chris transitioned HSM to a community social networking site.

Fifteen informants were recruited through personal contacts and participant led sampling. Participant led sampling was appropriate as it extended the sample frame to a more specific population of individuals transitioning their drinking behaviour. Two key elements of the HSM model are that individuals choose to voluntarily pledge abstinence from alcohol for a period of time (3, 6 or 12 months) and blog about their alcohol reduction practices and experiences. The pledge is designed to propel action towards reducing alcohol consumption; yet abstinence is not mandatory. Of the 15 informants, 12 chose to drink less alcohol with three informants abstaining. All informants blogged their thoughts, feelings, experiences, and alcohol reduction practices; with $80 \%$ reporting as active bloggers and the remaining occasionally blogging. Occasional bloggers blogged at least once a fortnight yet would actively scan HSM blogging activity at least every other day. Alcohol reduction was defined as practices associated with lowering alcohol consumption, including but not restricted to abstinence. Informants ranged from 22 to 36 years, self-reported high levels of alcohol consumption and represented a diverse range of socio-economic status

To meaningfully study the learning of alcohol behaviour change various forms of data were collected over a twelve month period. First, a netnographic method enabled an interpretive approach to explore the lived experiences of HSMers transitioning from excessive to more moderate levels of drinking (Kozinets, 2006). Textual and visual materials, primarily blogs, were collected and analysed. These data added richness to findings of indepth interviews which generated a perspective on alcohol reduction action, activities and interactions. Informants participated in a series of interviews depending on the length of their HSM journey; typically interviews occurred monthly. Interviews ranged from 50 to 120 minutes, were recorded and transcribed verbatim. 


\section{HSM as a consumption community}

\section{Learning to engage}

Learning to engage represents the transitioning of thoughts, emotions and psyche towards being a responsible drinker. Aligning new identity, shedding old identity and engaging in a reflexivity about alcohol and society is a critical aspect of creating alcohol reduction competency through learned socialisation.

Learning to break habits: first stop mind space!

Establishing new habits is critical to the HSM journey. Shedding ritualised habits involves learning how to live and socialise without alcohol. Informant's narratives constructed their previous practices of drunkenness as a necessary aspect of socialising and 'being with friends' with benefits positioned as 'fun', 'pleasure', 'being happy' and 'feeling good'. The nexus between drinking as a practice embedded in a wider drinking culture and the desire to drink less yet also socialise within alcohol fuelled environments represents a conflicting and challenging reality. Based on interviews removing oneself from alcohol environments was not a solution to enacting behaviour change. Rather HSMers chose to find ways to integrate within alcohol infused spaces while maintaining their commitment. Pete highlights 'socialising with alcohol is what we used to do and not socialising with my friends is not an option so I have to work out a way that I can do both'. He further illustrates the struggles and challenges of deciding not to drink or drinking less is a critical aspect of the HSM commitment:

Pete: 'HSM is not something you do for a couple of weeks, you commit for at least 3 months, up to 12 months. That's a long time. The challenge is finding a way to fit 
HSM in your life which means creating new ways of living without alcohol. HSM is a personal journey that you do with other people. You can't hide for 12 months. It's about making alcohol less important and not the reason why you socalise.'

For all informants joining HSM was about finding a good balance in maintaining the 'fun' and positive experiences with consuming less alcohol, alongside the challenges of doing HSM when peers asserted the dominant norm of drinking. Megan mentions:

"I think about alcohol all the time. Breaking this cycle is challenging! It's about enjoying the moment and making every moment count rather than thinking about what you don't have."

The period of time prior to committing to HSM was described in terms of emotional and psychological anguish as individuals questioned their survival without alcohol. Katy describes her pre-HSM anguish in terms of deciding on her personal mode of alcohol reduction.

Katy: I'm battling between two approaches to drinking. Moderation equals the ability to still be able to get pleasure from wine, but risks falling into bad habits. Abstinence equals control and commitment to a lifestyle that should bring me peace but feels boring, painful and going against what I have known for 15 plus years. Arghhh.

Veteran HSMers acknowledge this stage of reconciling alcohol reduction goals and interpreting the meaning of these goals to the self as a critical step towards commitment. There are no rules associated with giving up alcohol; rather the process requires individuals to personify and negotiate the new behaviour. Discourses revealed breaking habits requires a reflexivity regarding interactions in social contexts without alcohol involving cognitive, 
psychological and emotional repositioning of the self in relation to practices of sociality and dealing with social pressures to drink.

\section{Learning boundaries: creating personal meaning}

A necessary part of breaking habits is the creation of personal meaning around responsible drinking prior to the ‘doing’ of responsible drinking. Informants contextualised personal boundaries for enacting responsible drinking which were largely adapted from knowledge of safe drinking guidelines. For example, 'committing to my pledge - abstaining', 'one or two drinks on a Friday and Saturday night, nothing during the week' or 'two or three drinks when out with friend on weekends'. Others were less committal specifying their alcohol reduction boundary as 'banning drinking at home' yet not specifying drinking boundaries when socialising or as 'not denying myself alcohol but mindful of making efforts to reduce consumption'. Choosing to drink less alcohol, rather than completely abstain, was based on informant's 'liking of alcohol', 'not being able to visualise myself in a social situation without alcohol,' and the 'thought of completely banning alcohol as unrealistic'. For Steven, setting alcohol reduction boundaries was challenging illustrating his personal struggle to resist alcohol.

Steven: 'I'm customising my journey. My ultimate goal is not to be problem drinker. I want to be able to drink socially and not get drunk. I'm doing a 3 month ban, and then I'm on a self-imposed responsible drinking contract. I'm trying moderation - no more than 2-4 standard drinks per drinking session and no more than two separate drinking sessions per week. If I can make moderation work it's the ultimate goal but if I'm fooling myself and I do binge I'll go back to complete abstinence.'

\section{Learning to imagine}


The second learning mode of the CoP framework is imagination. Imagination refers to the construction of belonging to a community and construction of the self as a community member. Imagination represents the link between 'doing' of alcohol reduction practices in social contexts and the blogging about the trials and tribulation of experiences within the HSM community.

\section{Learning new consumption consistencies}

Creating new consumption consistencies involves learning, acquisition, and tailoring of alcohol reduction practices. Learning new alcohol reduction practices, while not easily verbalised, was viewed as more than simply limiting consumption. Annabelle describes her exploration and tailoring of alcohol reduction practices as 'illuminating', 'stressful', 'confronting' and 'emotional', both personally and socially. Reflecting on learning new alcohol reduction practices informants described the process of giving up as akin to 'putting on a new skin; you're undressing and finding a new way of living without alcohol' and as a series of new experiences with practices tailored to suit the individual and social circumstances. Yet, over time 'the new skin transformed into a comfy coat' as competency in alcohol reduction practices strengthened. For example, socialising in clubs or pubs informants engaged in a variety of alcohol reduction practices ranging from alcohol imitation strategies of 'drinking soda from a champagne glass', 'drinking mocktails' or 'soft drinks' to constraint strategies such as 'slowing down my drinking; holding onto my drink for longer', 'turning up to the pub late' 'swapping a full beer for an almost empty one', or 'alternating between alcoholic and non-alcoholic drinks'. Catching up with friends informally (e.g. BBQs or dinners at home) informants would bring their own 'packaged fruit juices, soft drinks, nonalcoholic wines' or as Isabelle explains their own creations: 
Isabelle: 'I really tested the limits one night when I turned up to dinner with freshly made cucumber/celery and ginger juice. Yes, I bought a juicer this week because I'm already sick of drinking soda water!'

Learning new consumption consistencies involves the tailoring of alcohol reduction practises, alongside control of when and how often to socialise. Narratives illustrate informants were not willing to totally eschew alcohol infused environments or not socialise with their friends. Nonetheless, informants explained that until they gained competency in their alcohol reduction skills they controlled their socialisation interactions. New rituals such as taking up 'running in the evening', 'going to the gym more often' allowed informants to not think about alcohol and drinking, particularly at evening time and weekends. As Craig mentions 'It's said that you don't ever break habits, you replace them with other things, so I guess more exercise has been my replacement - and it's great'. Learning new consumption practices illustrates self-control, imagination and creativity in the customisation of new rituals and alcohol reduction habits.

\section{Learning responsible drinking as a shared repertoire}

Sharing alcohol reduction experiences within the HSM community facilitates a 'we-ness' in learning and appropriating alcohol reduction as a consumption entity (Bagozzi and Dholakia (2002). As Megan explains the 'sense of communal participation starting from a feeling that we are all going through the process and that we all know - or potentially know - each other is what makes this work'. Similarly, Simon reflects 'it's that connectedness with others and the collective action we're all making towards creating a better future'. For Sally and Jackson, blogging and sharing alcohol reduction experiences is more than just giving up 
alcohol. Sally comments: 'People support you, give you tips. Even when you relapse you're not condemned. You get back on the bicycle and try again'. Likewise, Jackson explains:

“It's so great to have a forum where I can actually be honest about my drinking and affirming to find other nice humans who are battling the same stuff, being so frank and so real about how difficult it can be, but at the same time so encouraging of one another. Even though it's all in cyberspace this still feels like (and is) a living and breathing community... which means I also feel accountable to you, my dear HSMers”.

This discourse reinforces learning alcohol reduction practises is a continual negotiation of trial and error. Critically learning new alcohol consumption consistencies relies on a sociality of consumption and discursive validation of experiences.

\section{Learning to align}

The third learning mode of the CoP framework is alignment. Alignment refers to competency of individuals in developing and articulating a shared purpose, norms and codes of behaviour, and how these are transmitted beyond the community within the broader social landscape.

\section{Towards a heightened consciousness}

Narratives revealed learning and negotiating alcohol reduction practices requires not just behavioural action, but also reconciliation of the self. Informants constantly defined and redefined their identities and their desired identity as they travelled through the HSM journey. Narratives illustrate the departure from alcohol as an object of desire and sociability evidenced in the early stages of HSM, with thoughts pre-occupied with struggles and challenges of practising alcohol reduction and the fear of missing out by not drinking. As 
informants gained competency in their alcohol reduction practices, learning transitioned beyond a behavioural outcome (drinking less) towards a revitalisation of the self with life; achieved by setting higher order goals. Mike illustrates: You'd think that by banning booze I'd be limiting myself; limiting what I could do and all that. But it's exactly the opposite... removing alcohol has done quite the opposite. Transcending towards his future possible self, Mike has reinvigorated his love for climbing and is preparing to climb Kilimanjaro. Other HSMers contextualised their future possible self as 'studying for a pilot's licence', 'being there for my family', 'love my work not just survive it', 'living in Italy for six months', 'saving up to build my dream house', 'not working to live, taking time out to think about where I want to be when I'm 50 or 60'. Tim reconciles:

"I realised half way through doing HSM that I was doing it to give my life a new purpose. It's not just about the here and now and what pub we're going to tonight. My thoughts and ideas aren't blurred by alcohol. My life has a new direction. Definitely taking a longer term view rather than only thinking about what pub we're going to tonight.”

\section{Legitimising responsible drinking}

Responsible drinking for many is a new consumption entity. Narratives highlight informant's inscriptions of alcohol and society where 'not drinking isn't something that you considered'. As Keiran comments, 'HSM doesn't tell you to stop drinking but allows you a choice between not drinking and drinking'. Discourse highlighted the street-cred appeal of HSM in creating a space where taking time out from drinking is legitimised. Katie explains:

'My friends and I know drinking too much is bad, unhealthy, and we're tired of waking up with a hangover. But it's what you do, it's what the group does, so you do it to. Ads tell us not to drink so much and you think about not drinking but it doesn't 
get much further than that. HSM isn't big brother. It started from someone's personal journey and a reality that not drinking is hard, it's challenging emotionally and downright confronting. Reading about HSM I realised that I might be able to actually do something about my drinking and that I'm not doing it alone”.

Discourse highlights the value of HSM as a community of consumption where learning responsible drinking is continually interpreted and reinterpreted, and how the assertion of new norms and values challenges dominant inscriptions associated with Australia’s drinking culture. Niray explains:

"I find people are interested and fascinated by my decision not to drink alcohol for 3 months. I'm talking much more about why I decided to embark on this challenge and I'm realising that I'm making an impact on my friends drinking. Some have decided to join HSM, others are in that pre-stage of thinking about doing HSM, others totally think it's not normal not to drink.”

This excerpt illustrates the raising of responsible drinking within a discursive consciousness among friendship groups and the power of individual influence to share and propagate knowledge of alcohol reduction practices within broader society. Informants narratives highlight the HSM journey enabled a space for them to reflect on their ongoing relationship with alcohol, to critique the role of alcohol as an important social artefact, and more broadly to consider ways to address the cultural embed of alcohol in society. Leisa identifies "I didn't realise how attached I was to alcohol and how important it was in my life, to my friends and the extent it facilitates socialising”. Similarly, Niray notes “there's a lot of cheap wine on the market and I'm noticing alcohol advertising much more than before”. Megan has taken on 
the challenge to transfer as many people as he can to HSM as a 'way of getting my friends to think about how much they're drinking... it's my personal crusade to help change society”.

\section{Discussion and Implications}

The application of Wenger’s CoP framework to HSM, a mechanism to disrupt alcohol consumption habits, shows how learning of alcohol reduction practices and ultimately behaviour change can be understood as a situated social practice. Importantly for social marketing practice, knowledge creation and knowledge transfer of responsible drinking practice and meaning occurs as a relational interaction between individuals within a broader market system. Learning and knowledge creation occur through participation, where participation is denoted as meaningful activity developed through relationships, shared identity and socialisation of responsible drinking practices. The knowledge embedded in the HSM community approach to alcohol behaviour change supersedes any one individual, but rather results from the continual creation, interpretation and reinterpretation across many individuals within the community (Wenger, 1998). Individuals act as both individual consumers and collective citizens in the process of learning and engaging in meaningful socially-embedded everyday discourses and practices relating to alcohol reduction (Jackson, 2005).

Governments struggle with effecting alcohol-related cultural change precisely because of tensions between the nature of alcohol's pleasures and damage caused (Moore, 2010) and the cultural embed embracing an intoxigenic experience economy (Brain, 2000). In reality drinking and drunkenness is inextricably linked with community and cultural norms, yet social marketing for alcohol largely neglects the extent to which alcohol and its consumption is bound up in and complicated by emotional, symbolic and social meanings (Peattie and Peattie, 2009). Sociological treatments of behaviour change have relevance for social 
marketing thinking, particularly in incorporating social networks of influence to disrupt entrenched habits. Breaking entrenched habits, creating new norms and developing social marketing interventions that are of value and relevance to target audiences is critical in the delivery of desired social improvement.

As evidenced in this study responsible drinking is constructed as a distinct consumption entity, rather than viewed simply as being oppositional to risky drinking. This viewpoint allows for a broadening of the theoretical scope for developing social marketing solutions. Observing behaviour change as a human experience and as a distinct consumption entity permits social marketing thinking to extend beyond an individualistic perspective and be more inclusive of contemporary marketing paradigms, such as value co-creation, sustainable marketing, relationship marketing, and consumer culture theory to name but a few. As Lefebvre (2012) notes the future of social marketing centres on creating mutually satisfying exchanges, and as such the discipline must extend its current myopic perspective.

The alcohol reduction stories narrated in this study evidence how structural interventions connect individuals, who may not necessarily have connected with one another, to embed sustainable drinking practices into everyday life. HSM operates as a community of consumption, a service entity, and as a space where influencers (persons/organisations) interact with enactors (a person) involved in the behaviour change process (Russell-Bennett et al., 2013). Viewing the potential of the nexus between upstream (policy/institutional/ regulation) and downstream (individualised psychology/consumer behaviour) strategies a community approach to disrupting entrenched habits presents an opportunity to facilitate mutually satisfying exchanges. There is significant potential for mid-stream thinking, through design architecture, to disrupt and recreate contexts and structures to facilitate the sociality of new habits and behaviours. Critically, addressing behaviour change solutions from a midstream approach captures insight into how institutional structures impact the doing of 
behaviour change and how individuals as active participants in the behaviour change process transition from risky to less risky behaviours.

The findings from this study are qualitative and, as such, cannot be generalised. Exploration of issues associated with the structure and design of virtual behaviour change communities of consumption are outside the scope of this study. Additionally, the objective of this study was not to analyse the effectiveness of HSM in facilitating responsible drinking behaviour, but rather examine learning of responsible drinking as a social artefact. The emergence of alcohol behaviour change communities in Australia, including Febfast, Ocsober and Dry July, over the past 5 years each offering a variety of alcohol reduction models (e.g.: online, offline, fund raising) provides opportunity to examine alcohol reduction as a social phenomenon. To what extent do varying design formats affect learning and sustaining of alcohol reduction practices? What design concepts from other fields beyond Wenger's model can be applied? Are communities of behaviour change applicable to other risky behaviours? To what extent does market readiness affect success of creating new social norms related to positive behaviours?

Integration of 'healthy lifestyles' as a valid consumption choice within contemporary culture is a relatively new consumption paradigm. Inspired by Weibe’s (1952) suggestion that as a social good "brotherhood” could be sold using commercial marketing strategies, social marketing has gained currency as a good fit with government's growing political emphasis on behavioural change as the driver for health improvement (Peattie and Peattie 2009). Yet, as recent scholarship contends the future success of social marketing relies on a broader holistic view of behaviour change that is inexplicitly integrates the sociality of consumption in transforming behaviour. This study contributes significantly to this emerging debate in social marketing scholarship viewing behaviour change as a consumption entity and social artefact within a broader market system. 


\section{References}

Andreasen, A. (1995), Marketing social change, Jossey-Bass Publishers, San Francisco.

Ardichvilli, A., Page, V., and Wentling, T. (2003), "Motivation and barriers to participation in virtual knowledge-sharing communities of practice", Journal of Knowledge Management, Vol. 7 No. 1, pp. 64-77.

Bagozzi, R.P., and Dholakia, U.M. (2002), “Intentional social action in virtual communities”, Journal of Interactive Marketing, Vol. 16 No. 2, pp. 2-21.

Brown, J.S. and Duguid, P. (1998), “Organizing knowledge”, California Management Review, Vol. 40, pp. 90-111.

Donovan, R. (2011), “Social marketing’s mythunderstandings”, Journal of Social Marketing, Vol. 1 No. 1, pp. 8-16.

French, J., Spotswood, F., Tapp, A. and Stead, M. (2012), “Some reasonable but uncomfortable questions about social marketing”, Journal of Social Marketing, Vol. 2 No. 3, pp. 163-175.

Fry, M.L. (2011), “Discourses of consumer’s alcohol resistance identities”, Journal of Nonprofit and Public Sector Marketing, Vol. 23 No. 4, pp. 348-366.

Fry, M.L. (2010), “Countering consumption in a culture of intoxication”, Journal of Marketing Management, Vol. 26 No. 13, pp. 1270-1294.

Hagel. J. III \& Armstrong, A. (1997). Net Gain, Harvard Business School Press, Boston.

Hamley, B., \& Carah., N. (2012). One Sunday at a time: Evaluating Hello Sunday Morning. Foundation for Alcohol Research and Education, FARE, Canberra.

Handley, K., Sturdy, A., Fincham, R. and Clark, T. (2006), "Within and beyond communities of practice: Making sense of learning through participation, Identity and practice”, Journal of Management Studies, Vol, 43 No. 3, pp. 641-653.

Hoek, J. and Jones, S. (2011), "Regulation, public health and social marketing: A behaviour change trinity”, Journal of Social Marketing, Vol. 1 No. 1, pp. 32-44.

Jackson, T. (2005), Motivating sustainable consumption: A review of evidence on consumer behaviour and behavioural change, Sustainable Development Research Network, Guildford, UK.

Kozinets, R.V. (1999), "E-tribalized marketing?: the strategic implications of virtual communities of consumption”, European Management Journal, Vol. 17 No. 3, pp: 252264.

Kruglanski, A. W., Shah, J. Y., Fishbach, A., Friedman, R., Chun, W. Y., and SleethKeppler, D. (2002), “A theory of goal systems”, in Zanna, M.P. (Ed.), Advances in experimental social psychology, Academic Press, San Diego, CA, pp. 331-378. 
Lefebvre, C. (2012), “Transformative social marketing: Co-creating the social marketing discipline and brand”, Journal of Social Marketing, Vol.2 No.2, pp.118-129.

Lefebvre, C. (2011), ‘An integrative model for social marketing”, Journal of Social Marketing, Vol. 1 No. 1, pp.54-73.

Muniz, A.M. \& O’Guinn, T.C. (2001), “Brand community”, Journal of Consumer Research, Vol. 27 No. 4, pp. 412-432.

Peattie, K. \& Peattie, S. (2009), “Social marketing: A pathway to consumption reduction?”, Journal of Business Research, Vol. 62, pp. 260-268.

Richetin, J., Connor, M., \& Perugini, M. (2011), "Not doing is not the opposite of doing: Implications for attitudinal models of behavioural prediction”, Personality and Social Psychology Bulletin, Vol. 37 No. 1, pp. 40-54.

Russell-Bennett, R., Wood, W., and Previt, J. (2013), "Fresh ideas: Services thinking for social marketing”, Journal of Social Marketing, Vol. 3 No. 2, pp. 223-238.

Schau, H.P., Muniz, A.M., and Arnould, E.J. (2009), "How brand community practices create value”, Journal of Marketing, Vol 73, pp. 30-51.

Shanker, A., Elliott, R., \& Fitchett, J.A. (2009), "Identity, consumption and narratives of socialization”, Marketing Theory, Vol. 9, pp. 75-79.

Szmigin, I., Bengry-Howell, A., Griffin, C., Hackley, C. \& Mistral, W. (2011), “Social marketing, individual responsibility and the "culture of intoxication"”, European Journal of Marketing, Vol. 45 No. 5, pp. 759-79.

Verplanken, B. \& Wood, W. (2006), "Interventions to break and create consumer habits”, Journal of Public Policy and Marketing, Vol. 25 No. 1, pp. 90-103.

Weibe, G.D. (1951), “Merchandising commodities and citizenship on television”, Public Opinion Quarterly, Vol. 15 No. 4., pp. 679-691.

Wenger, E. (2000), “Communities of practice and social learning systems”, Organization, Vol. 7 No. 2, pp. 225-246.

Wenger, E. (1998), Communities of practice: Learning, meaning and identity, Cambridge: Cambridge University Press.

Wood, W. and Neal, D.T. (2007) "A new look at habits and the habit-goal interface”, Psychological Review, Vol. 114 No. 4, pp. 843-863.

Wymer, W. (2011), “Developing more effective social marketing strategies”, Journal of Social Marketing, Vol. 1 No. 1, pp. 17-31. 